\title{
Non-convex sweeping processes involving maximal monotone operators
}

\author{
Samir Adly a and Ba Khiet Le ${ }^{b}$ \\ ${ }^{a}$ XLIM UMR-CNRS 7252, Université de Limoges, Limoges, France; ${ }^{b}$ Centro de Modelamiento Matemático (CMM), \\ Universidad de Chile, Santiago, Chile
}

\section{ABSTRACT}

By using a regularization method, we study in this paper the global existence and uniqueness property of a new variant of non-convex sweeping processes involving maximal monotone operators. The system can be considered as a maximal monotone differential inclusion under a control term of normal cone type forcing the trajectory to be always contained in the desired moving set. When the set is fixed, one can show that the unique solution is right-differentiable everywhere and its right-derivative is rightcontinuous. Non-smooth Lyapunov pairs for this system are also analysed.

\section{KEYWORDS}

Sweeping process; differential inclusion; variational analysis; maximal monotone operator; non-smooth Lyapunov pairs

\section{Introduction}

Sweeping processes were proposed and thoroughly studied by Moreau in the seventies [1-4], which can be written as follows

$$
\left\{\begin{array}{l}
\dot{x}(t) \in-N_{C(t)}(x(t)) \text { a.e. } t \in[0, T], \\
x(0)=x_{0} \in C(0),
\end{array}\right.
$$

where $N_{C(t)}(\cdot)$ denotes the normal cone operator of the convex set $C(t)$ in the sense of convex analysis in a Hilbert space $H$. Existence and uniqueness of solutions of such systems and their classical variants (subjected to perturbation forces, prox-regular state dependent set $C(t, x)$, second-order sweeping processes ...) have been considered by many authors in the literature (see, e.g. [5-10]). In this paper, we are interested in a variant of sweeping processes associated with maximal monotone operators of following form

$$
\left\{\begin{array}{l}
\dot{x}(t) \in-A x(t)-N_{C(t)}(x(t)) \text { a.e. } t \in[0, T], \\
x(0)=x_{0},
\end{array}\right.
$$

where $A: H \rightrightarrows H$ is a set-valued maximal monotone operator and $N_{C(t)}(\cdot)$ denotes the proximal normal cone operator of the closed, prox-regular set $C(t)$ in Hilbert space $H$. The system (1) can be considered as a maximal monotone differential inclusion under control term $u(t) \in N_{C(t)}(\cdot)$ which guarantees that the trajectory $x(t)$ always belongs to the desired set $C(t)$ for all $t \in[0, T]$. When $C(t) \equiv C$ is a fixed, closed and convex subset of $H$, under some qualification conditions, e.g.

$$
C \cap \operatorname{int}(\operatorname{dom} A) \neq \emptyset \text { or } \operatorname{int}(C) \cap \operatorname{dom} A \neq \emptyset,
$$




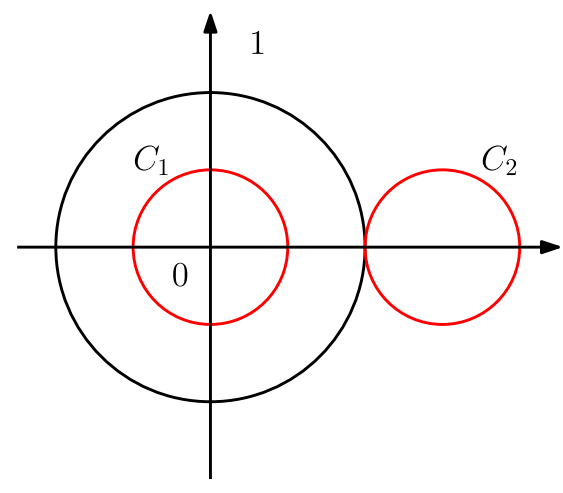

Figure 1. Counter-example for the prox-regular case.

then $A(\cdot)+N_{C}(\cdot)$ is a maximal monotone operator [11] and hence the existence and uniqueness of solutions can be obtained [12]. However, the situation is different when the closed set $C$ is assumed to be prox-regular instead of being convex. For example, let $H=\mathbb{R}^{2}$ and define $B: \mathbb{R}^{2} \rightarrow \mathbb{R}^{2},(x, y) \mapsto$ $(y,-x)$. It is easy to see that $B$ is a single-valued, continuous and monotone mapping with $\operatorname{dom}(B)=$ $\mathbb{R}^{2}$. Hence $B$ is maximal monotone. Let $A=B+N_{\mathbb{B}}$ where $\mathbb{B}=\mathbb{B}(0,1)$ denotes the closed unit ball in $\mathbb{R}^{2}$. Clearly, $A$ is maximal monotone with $\operatorname{dom}(A)=\mathbb{B}$. Let $C_{1}=\mathbb{B}(0,1 / 2)$ and $C_{2}=\mathbb{B}(3 / 2,1 / 2)$. The sets $C_{1}$ and $C_{2}$ are closed and convex. Thus $C=C_{1} \cup C_{2}$ is prox-regular (see Section 2) and $\operatorname{int}(C) \cap \operatorname{int}(\operatorname{dom} A) \neq \emptyset$. However, it is not difficult to see that the system

$$
\left\{\begin{array}{l}
\dot{x}(t) \in-A x(t)-N_{C}(x(t)) \text { a.e. } t \in[0, T] \\
x(0)=x_{0}=(1,0)
\end{array}\right.
$$

has no absolutely continuous solutions. Indeed, if the differential inclusion (3) has an absolutely continuous solution $x(\cdot)$, then this solution must be a constant function $x(\cdot) \equiv x_{0}$. The fact that $0 \notin A\left(x_{0}\right)+N_{C}\left(x_{0}\right)$ constitutes a contradiction (Figure 1).

Consequently, for problem (1) involving prox-regular set $C$, one needs more than the classical qualification condition (2) to ensure the existence of solutions. It is natural here to suppose that $C \subset \operatorname{int}(\operatorname{dom} A)$. If $A$ coincides with the subdifferential of a proper, lower semi-continuous and convex function $\varphi: H \rightarrow \mathbb{R} \cup\{+\infty\}$, i.e. $A \equiv \partial \varphi$, then the global existence and uniqueness of solutions are guaranteed. This is a particular case of evolution associated with primer lower nice function studied in [13]. For general $A$ and $C(t)$, if $x_{0} \in C(0) \cap \operatorname{int}(\operatorname{dom} A)$, we can prove the existence and uniqueness of local solutions (see Proposition 3.5). The global result can be obtained if

$$
\text { rge } C:=\bigcup_{0 \leq t \leq T} C(t) \subset \operatorname{int}(\operatorname{dom} A),
$$

and $A^{0}$ (the minimal norm operator of $A$ ) is linearly bounded on $C(t)$, i.e. $\left\|A^{0} x\right\| \leq \alpha(t)\|x\|+\beta(t)$ for all $x \in \operatorname{dom}(A)$, a.e. $t \in[0, T]$ for some $\alpha(\cdot), \beta(\cdot) \in L^{2}\left([0, T] ; \mathbb{R}_{+}\right)$(see Theorems 3.2 and 3.3).

For the particular case when the moving set $C(\cdot)$ is expanded, i.e. $C(s) \subset C(t)$ for all $t \geq s \geq 0$, and $A=\partial \varphi$ with $\varphi: H \rightarrow \mathbb{R} \cup\{+\infty\}$ proper, lower semi-continuous and convex function then the global well-posedness result, Lyapunov stability and some asymptotic behaviours of solutions are provided without the linear growth condition for $A^{0}$ (see Theorem 3.6). To the best of our knowledge, there have been no results about Lyapunov stability for general sweeping processes, which is known to be a hard problem. When $C(\cdot) \equiv C$ is fixed, one can show that the unique solution $x(\cdot)$ is right differentiable everywhere and $\dot{x}^{+}(\cdot)$ is right continuous. Then some criteria for non-smooth Lyapunov pairs for such system are given. 
The paper is organized as follow. We recall some basic notations, definitions and results which are used throughout the paper in Section 2. In Section 3 the existence and uniqueness of solutions of (1) are proved. In Section 4, we study some regular properties of solutions when $C(t) \equiv C$ and give criteria to weakly lower semi-continuous Lyapunov pairs by using proximal analysis. Section 5 ends the paper and contains some concluding remarks.

\section{Notations and preliminaries}

Let us begin with some notations used in the paper. Let $H$ be a Hilbert space. Denote by $\langle\cdot, \cdot\rangle,\|\cdot\|$ the scalar product and the corresponding norm in $H$. Denote by $I$ the identity operator, by $\mathbb{B}$ the unit ball in $H$ and $\mathbb{B}_{r}=r \mathbb{B}, \mathbb{B}_{r}(x)=\mathbb{B}(x, r)=x+r \mathbb{B}$. The distance from a point $s$ to a closed set $C$ is denoted by $d(s, C)$ or $d_{C}(s)$ and

$$
d(s, C):=\inf _{x \in C}\|s-x\| .
$$

The Hausdorff distance between two sets $C_{1}, C_{2}$ is defined by

$$
d_{H}\left(C_{1}, C_{2}\right):=\max \left\{\sup _{x_{1} \in C_{1}} d\left(x_{1}, C_{2}\right), \sup _{x_{2} \in C_{2}} d\left(x_{2}, C_{1}\right)\right\} .
$$

Denote by

$$
C^{0}:=\left\{c \in C:\|c\|=\inf _{c^{\prime} \in C}\left\|c^{\prime}\right\|\right\} \text { and }\|C\|:=\sup _{c \in C}\|c\| .
$$

It is known that if $C$ is closed and convex, then $C^{0}$ contains exactly one element. The set of all points in $C$ that are nearest to $s$ is denoted by

$$
\operatorname{Proj}(C, s):=\{x \in C:\|s-x\|=d(s, C)\} .
$$

When $\operatorname{Proj}(C, s)=\{x\}$, we can write $x=\operatorname{proj}(C, s)$ to emphasize the single-valued property. Let $x \in \operatorname{Proj}(C, s)$ and $t \geq 0$, then the vector $t(s-x)$ is called proximal normal to $C$ at $x$. The set of all such vectors is a cone, called proximal normal cone of $C$ at $x$ and denoted by $N^{P}(C, x)$. It is well-known $[14,15]$ that $\xi \in N^{P}(C, x)$ if and only if there exist some $\sigma>0, \delta>0$ such that

$$
\langle\xi, y-x\rangle \leq \delta\|y-x\|^{2} \text { for all } y \in C \cap \mathbb{B}_{\sigma}(x) .
$$

The regular-Fréchet normal cone $N^{F}(\cdot)$, the limiting Mordukhovich normal cone $N^{L}(\cdot)$ and the Clarke-convexified normal cone $N^{C}(\cdot)$ are defined, respectively, as follows

$$
\begin{gathered}
N^{F}(C, x):=\left\{\xi \in H: \forall \delta>0, \exists \sigma>0 \text { s.t. }\langle\xi, y-x\rangle \leq \delta\|y-x\| \text { for all } y \in C \cap \mathbb{B}_{\sigma}(x)\right\}, \\
\begin{array}{r}
N^{L}(C, x):=\left\{\xi \in H: \exists \xi_{n} \rightarrow \xi \text { weakly and } \xi_{n} \in N^{P}\left(C, x_{n}\right), x_{n} \rightarrow x \text { in } C\right\} \\
=\left\{\xi \in H: \exists \xi_{n} \rightarrow \xi \text { weakly and } \xi_{n} \in N^{F}\left(C, x_{n}\right), x_{n} \rightarrow x \text { in } C\right\}, \\
N^{C}(C, x):=\overline{\operatorname{co}} N^{L}(C, x) .
\end{array}
\end{gathered}
$$

If $x \notin C$, one has $N^{P}(C, x)=N^{F}(C, x)=N^{L}(C, x)=N^{C}(C, x)=\emptyset$ and for all $x \in C$ :

$$
N^{P}(C, x) \subset N^{F}(C, x) \subset N^{L}(C, x) \subset N^{C}(C, x) .
$$


If $C$ is prox-regular then these normal cones are coincide. In this case for simplicity, we can write $N(C, x)$.

Definition 1: The closed set $C$ is called $r$-prox-regular $(r>0)$ iff each point $s$ in the $r$-enlargement of $C$

$$
U_{r}(C):=\{w \in H: d(w, C)<r\},
$$

has a unique nearest point $\operatorname{proj}(C, s)$ and the mapping $\operatorname{proj}(C, \cdot)$ is continuous on $U_{r}(C)$.

Proposition 2.1 [10,16]: Let $C$ be a closed set in $H$ and $r>0$. The followings are equivalent

(1) $C$ is $r$-prox-regular.

(2) For all $x \in C$ and $\xi \in N^{L}(C, x)$ such that $\|\xi\|<r$, we have

$$
x=\operatorname{proj}(C, x+\xi)
$$

(3) For all $x \in C$ and $\xi \in N^{L}(C, x)$, we have

$$
\langle\xi, y-x\rangle \leq \frac{\|\xi\|}{2 r}\|y-x\|^{2} \quad \forall y \in C .
$$

(4) (Hypo-monotonicity) For all $x, x^{\prime} \in C, \xi \in N^{L}(C, x), \xi^{\prime} \in N^{L}\left(C, x^{\prime}\right)$ and $\xi, \xi^{\prime} \in \mathbb{B}$ we have

$$
\left\langle\xi-\xi^{\prime}, x-x^{\prime}\right\rangle \geq-\frac{1}{r}\left\|x-x^{\prime}\right\|^{2} .
$$

Let us recall the definition of proximal subgradient and asymptotic subgradient of a proper lower semi-continuous function by using proximal calculus.

Definition 2: Let $\varphi: H \rightarrow \mathbb{R} \cup\{+\infty\}$ be a proper lower semi-continuous function and $x \in H$ at which $\varphi$ is finite. One say that $\xi$ is a proximal subgradient of $\varphi$ at $x$, denoted by $\xi \in \partial_{P} \varphi(x)$ provided $(\xi,-1) \in N_{\text {epi } \varphi}^{P}(x, \varphi(x))$. And $\xi$ is called a singular subgradient of $\varphi$ at $x$, denoted by $\xi \in \partial_{\infty} \varphi(x)$ provided $(\xi, 0) \in N_{\text {epi } \varphi}^{L}(x, \varphi(x))$.

It is known that $\xi \in \partial_{\infty} \varphi(x)$ if and only if there exist sequences $\left(\alpha_{k}\right)_{k \in \mathbb{N}} \subset \mathbb{R}_{+},\left(x_{k}\right)_{k \in \mathbb{N}},\left(\xi_{k}\right)_{k \in \mathbb{N}}$ such that $\alpha_{k} \rightarrow 0^{+}, x_{k} \rightarrow_{\varphi} x, \xi_{k} \in \partial_{P} \varphi\left(x_{k}\right)$ and $\alpha_{k} \xi_{k} \rightarrow \xi$ (see, e.g. [17]).

In the following, we summarize some known definitions and results concerning maximal monotone operators. The domain and graph of a set-valued operator $A: H \rightrightarrows H$ are defined, respectively, by

$$
\operatorname{dom} A:=\{x \in H: A(x) \neq \emptyset\}, \quad \operatorname{gph} A:=\{(x, y): x \in H, y \in A(x)\} .
$$

The operator $A$ is called monotone if for all $x, y \in H, x^{*} \in A(x), y^{*} \in A(y)$, we have $\left\langle x^{*}-y^{*}, x-y\right\rangle \geq$ 0 . In addition, if there is no monotone mapping $B$ such that gph $A$ is contained strictly in gph $B$, then $A$ is called maximal monotone. When $A$ is a maximal monotone operator, the minimal norm operator $A^{0}$ of $A$ is a single-valued mapping defined by $A^{0}: H \rightarrow H, x \mapsto(A(x))^{0}$.

Proposition 2.2 [12]: Let $A: H \rightrightarrows H$ be maximal monotone and let $\lambda>0$. Then

(1) the resolvent of $A$ defined by $J_{\lambda}^{A}:=(I+\lambda A)^{-1}$ is a non-expansive single-valued map from $H$ to $H$.

(2) the Yosida approximation of $A$ defined by $A_{\lambda}:=\frac{1}{\lambda}\left(I-J_{\lambda}^{A}\right)=\left(\lambda I+A^{-1}\right)^{-1}$ satisfies

(i) for all $x \in H, A_{\lambda}(x) \in A\left(J_{\lambda}^{A} x\right)$,

(ii) $A_{\lambda}$ is Lipschitz continuous with constant $\frac{1}{\lambda}$ and maximal monotone.

(iii) If $x \in \operatorname{dom} A$, then $\left\|A_{\lambda} x\right\| \leq\left\|A^{0} x\right\|$, where $A^{0} x$ is the element of $A x$ of minimal norm.

(3) If $x_{\lambda} \rightarrow x$ and $\left(A_{\lambda} x_{\lambda}\right)_{\lambda}$ is bounded as $\lambda \rightarrow 0$ then $x \in \operatorname{dom} A$. Moreover, if $y$ is a cluster point of $\left(A_{\lambda} x_{\lambda}\right)_{\lambda}$ as $\lambda \rightarrow 0$ then $y \in A(x)$. 
Proposition 2.3 [12]: Let $A: H \rightrightarrows H$ be maximal monotone. Then

(1) A is sequentially weak-strong and strong-weak closed.

(2) A is locally bounded in int.dom $A$ ), the interior of $\operatorname{dom} A$.

(3) Let $T>0$. Define $\mathcal{A}: L^{2}([0, T] ; H) \rightrightarrows L^{2}([0, T] ; H)$ by

$$
y(\cdot) \in \mathcal{A} x(\cdot) \text { iff } y(t) \in A x(t) \text { a.e. } t \in[0, T] .
$$

Then $\mathcal{A}$ is also maximal monotone.

We finish this section with a version of Gronwall's inequality (see, e.g. Lemma 4.1 in [18]).

Lemma 2.4: Let $T>0$ be given and $a(\cdot), b(\cdot) \in L^{1}([0, T] ; \mathbb{R})$ with $b(t) \geq 0$ for almost all $t \in[0, T]$. Let the absolutely continuous function $w:[0, T] \rightarrow \mathbb{R}_{+}$satisfy

$$
(1-\alpha) w^{\prime}(t) \leq a(t) w(t)+b(t) w^{\alpha}(t), \quad \text { a.e. } t \in[0, T],
$$

where $0 \leq \alpha<1$. Then for all $t \in[0, T]$, one has

$$
w^{1-\alpha}(t) \leq w^{1-\alpha}(0) \exp \left(\int_{0}^{t} a(\tau) \mathrm{d} \tau\right)+\int_{0}^{t} \exp \left(\int_{s}^{t} a(\tau) \mathrm{d} \tau\right) b(s) \mathrm{d} s .
$$

\section{Existence and uniqueness of solutions}

In this section, we study the existence and uniqueness of solutions of (1) by using a regularization approach and some properties of a classical class of perturbed sweeping processes. First, let us make the following assumptions. Let $r>0$ be given.

Assumption 1: For each $t \in[0, T]$, the set $C(t)$ is non-empty closed and r-prox-regular.

Assumption 2: The mapt $\mapsto C(t)$ is absolutely continuous, i.e. there exist a non-decreasing function $v:[0, T] \rightarrow \mathbb{R}_{+}$with $v(0)=0$ such that for all $0 \leq s<t \leq T$, one has

$$
d_{H}(C(t), C(s)) \leq v(t)-v(s)
$$

Assumption 3: $\quad$ The set-valued mapping $A: H \rightrightarrows H$ is a maximal monotone operator.

One has the following lemma, which is a similar result to Proposition 1 in [6]. We give here a different proof, where the idea can be used later in the proof of Theorem 3.6.

Lemma 3.1: Assume that Assumptions 1, 2 are satisfied and $f: H \rightarrow H$ be a Lipschitz continuous function. Let $x(\cdot):[0, T] \rightarrow H$ be the unique solution of the differential inclusion (see, e.g. [6] for the existence and uniqueness of solutions)

$$
\left\{\begin{array}{l}
\dot{x}(t) \in-N_{C(t)}(x(t))+f(x(t)), \text { a.e. } t \in[0, T], \\
x(0)=x_{0} \in C(0)
\end{array}\right.
$$

Then for almost every $t \in[0, T]$, one has

$$
\|\dot{x}(t)-f(x(t))\| \leq \dot{v}(t)+\|f(x(t))\| .
$$

Proof: Fix some $t \geq 0$ such that $x(\cdot)$ is differentiable at $t$. From the $r$-prox-regularity of $C(t)$, one has

$$
\langle\dot{x}(t)-f(x(t)), c-x(t)\rangle \leq \beta\|c-x(t)\|^{2} \quad \forall c \in C(t),
$$


where $\beta:=\frac{\|\dot{x}(t)-f(x(t))\|}{2 r}$ (see Proposition 2.1). Let $\varepsilon>0$ be small enough. Since $x(t+\varepsilon) \in C(t+\varepsilon) \in$ $C(t)+(v(t+\varepsilon)-v(t)) \mathbb{B}$, there exist $c_{\varepsilon} \in C(t)$ and $y_{\varepsilon} \in \mathbb{B}$ such that $x(t+\varepsilon)=c_{\varepsilon}+(v(t+\varepsilon)-v(t)) y_{\varepsilon}$. Choosing $c=c_{\varepsilon}$ in (10), one has

$$
\begin{aligned}
\langle\dot{x}(t)-f(x(t)), x(t+\varepsilon)-x(t)\rangle \leq & (v(t+\varepsilon)-v(t))\left\langle\dot{x}(t)-f(x(t)), y_{\varepsilon}\right\rangle \\
& +\beta\left\|x(t+\varepsilon)-(v(t+\varepsilon)-v(t)) y_{\varepsilon}-x(t)\right\|^{2} .
\end{aligned}
$$

Dividing both sides by $\varepsilon$ and let $\varepsilon \rightarrow 0$, it implies that

$$
\langle\dot{x}(t)-f(x(t)), \dot{x}(t)\rangle \leq \dot{v}(t)\|\dot{x}(t)-f(x(t))\| .
$$

Hence

$$
\begin{aligned}
\|\dot{x}(t)-f(x(t))\|^{2} & \leq\langle\dot{x}(t)-f(x(t)), f(x(t))\rangle+\dot{v}(t)\|\dot{x}(t)-f(x(t))\| \\
& \leq\|\dot{x}(t)-f(x(t))\|(\|f(x(t))\|+\dot{v}(t)) .
\end{aligned}
$$

Then the result follows.

We start with a global existence and uniqueness result for problem (1). Let us denote the range of $C(\cdot)$ by rge $C$ defined as follows

$$
\operatorname{rge} C:=\bigcup_{t \in[0, T]} C(t)
$$

Theorem 3.2: Let Assumptions 1, 2 and 3 hold. Suppose that

(i) $\operatorname{rge} C \subset \operatorname{dom} A$;

(i) there exists $\alpha(\cdot), \beta(\cdot) \in L^{2}\left([0, T] ; \mathbb{R}_{+}\right)$s.t. $\left\|A^{0}(x)\right\| \leq \alpha(t)\|x\|+\beta(t) \forall x \in C(t)$, for a.e. $t \in[0, T]$.

Then for each initial condition $x_{0} \in C(0)$, there exists an absolutely continuous solution $x(\cdot)$ of problem (1) on $[0, T]$.

Proof: For each $\lambda>0$, denote $A_{\lambda}$ the Moreau-Yosida approximation of $A$. Then $A_{\lambda}: H \rightarrow H$ is $1 / \lambda$-Lipschitz continuous. We consider the following approximate sweeping process

$$
\left\{\begin{array}{l}
\dot{x_{\lambda}}(t) \in-A_{\lambda} x_{\lambda}(t)-N_{C(t)}\left(x_{\lambda}(t)\right) \text { a.e. } t \in[0, T], \\
x_{\lambda}(0)=x_{0} \in C(0) .
\end{array}\right.
$$

For each $\lambda>0$, the differential inclusion (12) has a unique solution $x_{\lambda}(\cdot)$ on $[0, T]$ (see, e.g. [6]). By Lemma 3.1, we have

$$
\left\|\dot{x}_{\lambda}(t)+A_{\lambda} x_{\lambda}(t)\right\| \leq\left\|A_{\lambda} x_{\lambda}(t)\right\|+\dot{v}(t) .
$$

Note that $\left\|A_{\lambda} x_{\lambda}(t)\right\| \leq\left\|A^{0} x_{\lambda}(t)\right\| \leq \alpha(t)\left\|x_{\lambda}(t)\right\|+\beta(t)$. Hence

$$
\left\|\dot{x}_{\lambda}(t)\right\| \leq 2\left\|A_{\lambda} x_{\lambda}(t)\right\|+\dot{v}(t) \leq 2 \alpha(t)\left\|x_{\lambda}(t)\right\|+2 \beta(t)+\dot{v}(t) .
$$

By using Gronwall's inequality, one obtains that

$$
\left\|x_{\lambda}(t)\right\| \leq x_{0} e^{a}+(2 b+v(T)) e^{a}:=M
$$

where $a:=\int_{0}^{T} \alpha(s) \mathrm{d} s, b:=\int_{0}^{T} \beta(s) \mathrm{d} s$. Let

$$
\xi(t):=M \alpha(t)+\beta(t) .
$$


Then $\xi \in L^{2}\left([0, T] ; \mathbb{R}_{+}\right)$and

$$
\left\|\dot{x}_{\lambda}(t)+A_{\lambda} x_{\lambda}(t)\right\| \leq \xi(t)+\dot{v}(t) \text { and }\left\|A_{\lambda} x_{\lambda}(t)\right\| \leq \xi(t) \text { a.e. } t \in[0, T] .
$$

Given some $\lambda, \mu>0$. From the prox-regular property of $C(t)$ and Proposition 2.1, one has for a.e. $t \in[0, T]$

$$
\left\langle\dot{x}_{\lambda}(t)+A_{\lambda} x_{\lambda}(t)-\dot{x}_{\mu}(t)-A_{\mu} x_{\mu}(t), x_{\lambda}(t)-x_{\mu}(t)\right\rangle \leq \frac{\xi(t)+\dot{v}(t)}{r}\left\|x_{\lambda}(t)-x_{\mu}(t)\right\|^{2} .
$$

Note that $A$ is a maximal monotone operator and

$$
x_{\lambda}(t)=J_{\lambda} x_{\lambda}(t)+\lambda A_{\lambda} x_{\lambda}(t) \text { with } A_{\lambda} x_{\lambda}(t) \in A\left(J_{\lambda} x_{\lambda}(t)\right) .
$$

Therefore,

$$
\begin{aligned}
\left\langle A_{\lambda} x_{\lambda}(t)-A_{\mu} x_{\mu}(t), x_{\lambda}(t)-x_{\mu}(t)\right\rangle= & \left\langle A_{\lambda} x_{\lambda}(t)-A_{\mu} x_{\mu}(t), J_{\lambda} x_{\lambda}(t)\right. \\
& \left.+\lambda A_{\lambda} x_{\lambda}(t)-J_{\mu} x_{\mu}(t)-\mu A_{\mu} x_{\mu}(t)\right\rangle \\
\geq & \left\langle A_{\lambda} x_{\lambda}(t)-A_{\mu} x_{\mu}(t), \lambda A_{\lambda} x_{\lambda}(t)-\mu A_{\mu} x_{\mu}(t)\right\rangle \\
\geq & -\frac{1}{4}\left(\lambda\left\|A_{\lambda} x_{\lambda}(t)\right\|^{2}+\mu\left\|A_{\mu} x_{\mu}(t)\right\|^{2}\right) \\
\geq & -\frac{1}{4}(\lambda+\mu) \xi^{2}(t) .
\end{aligned}
$$

From (15), we get

$$
\frac{\mathrm{d}}{\mathrm{d} t}\left\|x_{\lambda}(t)-x_{\mu}(t)\right\|^{2} \leq \frac{1}{2}(\lambda+\mu) \xi^{2}(t)+\frac{2(\xi(t)+\dot{v}(t))}{r}\left\|x_{\lambda}(t)-x_{\mu}(t)\right\|^{2} .
$$

Using Gronwall's inequality (Lemma 2.4) and noting that $x_{\lambda}(0)=x_{\mu}(0)=x_{0}$, one has for all $t \in[0, T]:$

$$
\begin{aligned}
\left\|x_{\lambda}(t)-x_{\mu}(t)\right\|^{2} & \leq \frac{1}{2}(\lambda+\mu) \int_{0}^{t} \exp \left(\int_{s}^{t} \frac{2(\xi(\tau)+\dot{v}(\tau))}{r} \mathrm{~d} \tau\right) \xi^{2}(s) \mathrm{d} s \\
& \leq \frac{1}{2}(\lambda+\mu) c_{2}^{2} e^{2\left(c_{1}+v(T)\right) / r},
\end{aligned}
$$

where

$$
c_{1}:=\|\xi(\cdot)\|_{L^{1}([0, T] ; H)} \text { and } c_{2}:=\|\xi(\cdot)\|_{L^{2}([0, T] ; H)} .
$$

Consequently,

$$
\left\|x_{\lambda}(t)-x_{\mu}(t)\right\| \leq \frac{1}{\sqrt{2}} c_{2} e^{\left(c_{1}+v(T)\right) / r} \sqrt{(\lambda+\mu)} \quad \text { for all } t \in[0, T]
$$

Hence $\left(x_{\lambda}(\cdot)\right)_{\lambda>0}$ is a Cauchy sequence in $C([0, T] ; H)$. As a consequence, there exists a function $x(\cdot) \in C([0, T] ; H)$ such that $x_{\lambda} \rightarrow x$ uniformly on $[0, T]$ as $\lambda \rightarrow 0$ and

$$
\left\|x_{\lambda}(t)-x(t)\right\| \leq \frac{1}{\sqrt{2}} c_{2} e^{\left(c_{1}+v(T)\right) / r} \sqrt{\lambda} .
$$

Using Proposition 2.2 and the fact that $\left\|A_{\lambda} x_{\lambda}(t)\right\| \leq \xi(t)$ for a.e. $t \in[0, T]$, one has $x(t) \in \operatorname{dom}(A)$ for a.e. $t \in[0, T]$. Furthermore, $x(t) \in C(t)$ because $x_{\lambda}(t) \in C(t)$ for all $\lambda>0$ and $C(t)$ is closed. 
Since $\left\|\dot{x}_{\lambda}(t)\right\| \leq 2 \xi(t)+\dot{v}(t)$ for almost all $t \in[0, T]$, there exists a sequence $\lambda_{n} \rightarrow 0$ such that $\dot{x}_{\lambda_{n}}$ converges weakly to some $v(\cdot) \in L^{2}([0, T] ; H)$. Classical arguments permit us to show that $\dot{x}=v$ (see, e.g. [12]). On the other hand, we have

$$
\left\|A_{\lambda_{n}} x_{\lambda_{n}}(t)\right\| \leq \xi(t) \quad \text { for a.e. } t \in[0, T]
$$

Hence, there exist a subsequence, still denoted by $\left(A_{\lambda_{n}} x_{\lambda_{n}}(\cdot)\right)_{n}$, and $\gamma(\cdot) \in L^{2}([0, T] ; H)$ such that $A_{\lambda_{n}} x_{\lambda_{n}}$ converges to $\gamma$ weakly in $\left.L^{2}([0, T] ; H)\right)$. Then we obtain

$$
\dot{x}_{\lambda_{n}}+A_{\lambda_{n}} x_{\lambda_{n}} \text { converges weakly to } \dot{x}+\gamma \text { in } L^{2}([0, T] ; H) \text {. }
$$

Using Mazur's lemma, for each $n$, there exist an integer $T(n)>n$ and real numbers $s_{k, n} \geq 0$ such that $\sum_{k=n}^{T(n)} s_{k, n}=1$ and $\sum_{k=n}^{T(n)} s_{k, n}\left(\dot{x}_{\lambda_{k}}+A_{\lambda_{k}} x_{\lambda_{k}}\right)$ converges strongly to $\dot{x}+\gamma$ in $L^{2}([0, T] ; H)$. Extracting a subsequence, we may suppose that

$$
\sum_{k=n}^{T(n)} s_{k, n}\left(\dot{x}_{\lambda_{k}}(t)+A_{\lambda_{k}} x_{\lambda_{k}}(t)\right) \rightarrow \dot{x}(t)+\gamma(t) \text { as } n \rightarrow+\infty,
$$

for all $t \in[0, T] \backslash N$ for some negligible set $N \subset[0, T]$. Fixed $t \in[0, T] \backslash N$, from the prox-regularity of $C(t)$, one has

$$
\left\langle\dot{x}_{\lambda_{k}}(t)+A_{\lambda_{k}} x_{\lambda_{k}}(t), y-x_{\lambda_{k}}(t)\right\rangle \geq-\frac{\xi(t)+\dot{v}(t)}{2 r}\left\|y-x_{\lambda_{k}}(t)\right\|^{2}, \quad \forall y \in C(t) .
$$

Hence,

$$
\begin{aligned}
\Lambda_{n}(t) & :=\sum_{k=n}^{T(n)} s_{k, n}\left\langle\dot{x}_{\lambda_{k}}(t)+A_{\lambda_{k}} x_{\lambda_{k}}(t), y-x_{\lambda_{k}}(t)\right\rangle \\
& \geq-\frac{\xi(t)+\dot{v}(t)}{2 r} \sum_{k=n}^{T(n)} s_{k, n}\left\|y-x_{\lambda_{k}}(t)\right\|^{2}, \quad \forall y \in C(t) .
\end{aligned}
$$

Note that since $x_{\lambda_{n}}(t) \rightarrow x(t)$ as $n \rightarrow+\infty$, we have

$$
\left|\sum_{k=n}^{T(n)} s_{k, n}\left\langle\dot{x}_{\lambda_{k}}(t)+A_{\lambda_{k}} x_{\lambda_{k}}(t), x(t)-x_{\lambda_{k}}(t)\right\rangle\right| \leq(\xi(t)+\dot{v}(t)) \sum_{k=n}^{T(n)} s_{k, n}\left\|x(t)-x_{\lambda_{k}}(t)\right\| \rightarrow 0 .
$$

Thus

$$
\begin{aligned}
\Lambda_{n}= & \sum_{k=n}^{T(n)} s_{k, n}\left\langle\dot{x}_{\lambda_{k}}(t)+A_{\lambda_{k}} x_{\lambda_{k}}(t), y-x(t)\right\rangle \\
& +\sum_{k=n}^{T(n)} s_{k, n}\left\langle\dot{x}_{\lambda_{k}}(t)+A_{\lambda_{k}} x_{\lambda_{k}}(t), x(t)-x_{\lambda_{k}}(t)\right\rangle \\
& \rightarrow\langle\dot{x}(t)+\gamma(t), y-x(t)\rangle \text { as } n \rightarrow+\infty .
\end{aligned}
$$

On the other hand

$$
\sum_{k=n}^{T(n)} s_{k, n}\left\|y-x_{\lambda_{k}}(t)\right\|^{2} \rightarrow\|y-x(t)\|^{2}
$$


since $x_{\lambda_{n}}(t) \rightarrow x(t)$ as $n \rightarrow+\infty$. Passing to the limit as $n \rightarrow+\infty$ in (20), we get

$$
\langle\dot{x}(t)+\gamma(t), y-x(t)\rangle \geq-\frac{\xi(t)+\dot{v}(t)}{2 r}\|y-x(t)\|^{2}, \quad \forall y \in C(t) .
$$

Therefore

$$
\dot{x}(t)+\gamma(t) \in-N_{C(t)}(x(t)) .
$$

Let us recall that

$$
A_{\lambda_{n}} x_{\lambda_{n}}(t) \in A\left(J_{\lambda_{n}} x_{\lambda_{n}}(t)\right) \text { for a.e. } t \in[0, T],
$$

and $A_{\lambda_{n}} x_{\lambda_{n}}$ converges weakly to $\gamma$ in $L^{2}([0, T] ; H)$. In addition $J_{\lambda_{n}} x_{\lambda_{n}}$ converges strongly to $x$ in $\left.L^{2}([0, T] ; H)\right)$ since

$$
\begin{aligned}
\left\|J_{\lambda_{n}} x_{\lambda_{n}}(t)-x(t)\right\| & \leq\left\|J_{\lambda_{n}} x_{\lambda_{n}}(t)-x_{\lambda_{n}}(t)\right\|+\left\|x_{\lambda_{n}}(t)-x(t)\right\| \\
& =\lambda_{n}\left\|A_{\lambda_{n}} x_{\lambda_{n}}(t)\right\|+\left\|x_{\lambda_{n}}(t)-x(t)\right\| \\
& \leq \lambda_{n} \xi(t)+\left\|x_{\lambda_{n}}(t)-x(t)\right\| \rightarrow 0 \text { as } n \rightarrow+\infty
\end{aligned}
$$

Consequently, by using Proposition 2.3, one has

$$
\gamma(t) \in A x(t) \text { for a.e. } t \in[0, T] .
$$

From (21) and (23), we deduce that

$$
\dot{x}(t) \in-A x(t)-N_{C(t)}(x(t)) \text { for a.e. } t \in[0, T],
$$

which completes the proof of Theorem 3.2.

Under some additional assumptions, we can prove a uniqueness result. The following theorem is in this sense.

Theorem 3.3: Let Assumptions 1-3 hold and suppose that

$$
\operatorname{rge} C \subset \operatorname{int}(\operatorname{dom} A) \text {. }
$$

Then, for given initial condition $x_{0} \in C(0)$, the problem (1) has at most one solution.

Proof: Let $x_{1}(\cdot), x_{2}(\cdot)$ be two solutions of (1) satisfying the initial conditions $x_{1}(0)=x_{2}(0)=x_{0}$. Since $A$ is locally bounded on rge $C$, there exists some $\rho>0$ and $K>0$ such that $A$ is bounded by $K$ on $\mathbb{B}\left(x_{0}, \rho\right)$. Note that $x_{1}(\cdot), x_{2}(\cdot)$ are continuous, then there exists some positive constant $T^{\prime}<T$ such that $x_{i}\left(\left[0, T^{\prime}\right]\right) \subset \mathbb{B}\left(x_{0}, \rho\right), i=1,2$. There exist $f_{i}(\cdot) \in-A x_{i}(\cdot)$ such that for a.e. $t \in\left[0, T^{\prime}\right]$

$$
\dot{x}_{i}(t) \in f_{i}(t)-N_{C(t)}\left(x_{i}(t)\right), \quad i=1,2 .
$$

Using a similar argument as in Lemma 3.1, one has

$$
\left\|\dot{x}_{i}(t)-f_{i}(t)\right\| \leq\left\|f_{i}(t)\right\|+\dot{v}(t) \leq K+\dot{v}(t) .
$$

From the monotonicity of $A$ and the prox-regularity of $C(t)$, one obtains

$$
\left\langle\dot{x}_{1}(t)-\dot{x}_{2}(t), x_{1}(t)-x_{2}(t)\right\rangle \leq \frac{K+\dot{v}(t)}{r}\left\|x_{1}(t)-x_{2}(t)\right\|^{2} \text {, a.e. } t \in\left[0, T^{\prime}\right] .
$$


Consequently,

$$
\frac{\mathrm{d}}{\mathrm{d} t}\left\|x_{1}(t)-x_{2}(t)\right\|^{2} \leq \frac{2(K+\dot{v}(t))}{r}\left\|x_{1}(t)-x_{2}(t)\right\|^{2}, \quad \text { a.e. } t \in\left[0, T^{\prime}\right]
$$

Using Gronwall's inequality, one has $\left\|x_{1}(t)-x_{2}(t)\right\| \leq 0$ for all $t \in\left[0, T^{\prime}\right]$, or equivalently $x_{1} \equiv x_{2}$ on $\left[0, T^{\prime}\right]$.

Suppose now that there exists $t_{1} \in[0, T]$ such that $x_{1}\left(t_{1}\right) \neq x_{2}\left(t_{1}\right)$. Let

$$
E:=\left\{t \in\left[0, t_{1}\right]: x_{1}(t) \neq x_{2}(t)\right\}
$$

Since $t_{1} \in E$ and $E$ is bounded from below, there exists $\alpha:=\inf E$ where $\alpha \in\left(0, t_{1}\right]$ and for all $t \in[0, \alpha): x_{1}(t)=x_{2}(t)$. By the continuity of $x_{1}(\cdot)$ and $x_{2}(\cdot)$, we have $x_{1}(\alpha)=x_{2}(\alpha)$ which implies that $\alpha<t_{1}$. With the same argument as above, there exists some $T^{\prime}>0$ such that $x_{1}(\cdot) \equiv x_{2}(\cdot)$ on $\left[0, \alpha+T^{\prime}\right]$. This constitutes a contradiction with the definition of $\alpha=\inf E$. Thus $x_{1}(\cdot) \equiv x_{2}(\cdot)$ on $[0, T]$.

Let us provide an example in parabolic variational inequalities.

Example 3.4: Let $\Omega$ be a bounded subset of $\mathbb{R}^{n}$ and $H:=\mathcal{L}^{2}(\Omega), U:=\mathcal{H}^{2}(\Omega) \cap \mathcal{H}_{0}^{1}(\Omega)$. Let be given $\psi \in \mathcal{L}^{2}(0, T ; U), M \in \mathcal{L}^{2}\left(0, T ; \mathbb{R}_{+}\right)$such that $\psi(\cdot)$ is $k$-Lipschitz continuous with respect to the supremum norm. For each $t \in[0, T]$, we define

$$
C(t):=C_{1}(t) \cup C_{2}(t)
$$

where

$$
C_{1}(t)=\{v \in U: v \geq \psi(t) \text { a.e. on } \Omega ;\|\Delta v\| \leq M(t)\}
$$

and

$$
C_{2}(t)=\{v \in U: v \leq \psi(t)-1 \text { a.e. on } \Omega ;\|\Delta v\| \leq M(t)\} .
$$

It is easy to see that for each $t$, the set $C(t)$ is closed, prox-regular (but non-convex) since $C_{1}(t), C_{2}(t)$ are two disjoint closed, convex sets and $\left\|v_{1}-v_{2}\right\| \geq \sqrt{m(\Omega)}$ for all $v_{1} \in C_{1}(t), v_{2} \in C_{2}(t)$, where $m(\Omega)$ is the volume of $\Omega$. Furthermore, $C(\cdot)$ is $k$-Lipschitz continuous since $\psi(\cdot)$ is $k$-Lipschitz continuous. Let be given $u_{0} \in C(0)$. We consider the following parabolic variational inequalities with a moving obstacle : find $u(t) \in C(t)$ such that $u(0)=u_{0} \in C(0)$ and for a.e. $t \in[0, T]$, there exists $\delta_{t}>0$ satisfying

$$
\int_{\Omega} \dot{u}(t)(v(t)-u(t)) \mathrm{d} x+\int_{\Omega} \nabla u(t) \cdot(\nabla v(t)-\nabla u(t)) \mathrm{d} x \geq-\delta_{t}\|v(t)-u(t)\|^{2}, \quad \forall v(t) \in C(t) .
$$

Let us define the operator $A: H \rightarrow H$ as $A:=-\Delta$, where $\Delta$ is the Laplace operator. Then $A$ is a self-adjoint maximal monotone operator with $\operatorname{dom}(A)=U$ and $\overline{\operatorname{dom}(A)}=H$ (see, e.g. [12,19]). It is easy to see that

$$
\int_{\Omega} A u(t)(v(t)-u(t)) \mathrm{d} x=\int_{\Omega} \nabla u(t) \cdot \nabla(v(t)-u(t)) \mathrm{d} x, \quad \forall u, v \in U .
$$

Then the problem (25) can be rewritten as follows

$$
\dot{u}(t) \in-A u(t)-N_{C(t)} u(t)
$$

and all the assumptions of Theorem 3.2 are satisfied. Thus, for $u_{0} \in C(0)$, there exists an absolutely continuous solution $u(\cdot)$ of (26), or equivalently, of (25). In addition, if $M(\cdot)$ is a constant function, by using Remark 1, one deduces the uniqueness of solutions. 
Remark 1: In Theorem 3.3, we can also relax the condition $\operatorname{rge} C \subset \operatorname{int}(\operatorname{dom} A)$ by the assumption that $A$ is locally bounded on rge $C$, i.e. for all $x \in \operatorname{rge} C$, there exists $K>0, \rho>0$ such that $A$ is bounded by $K$ in $\mathbb{B}\left(x_{0}, \rho\right) \cap$ rge $C$.

The following proposition gives us the local result requiring only $x_{0} \in C(0) \cap \operatorname{int}(\operatorname{dom} A)$.

Proposition 3.5: Let Assumptions 1-3 hold. If $x_{0} \in C(0) \cap \operatorname{int}(\operatorname{dom} A)$ then problem (1) has a unique local solution.

Proof: Since $x_{0} \in \operatorname{int}(\operatorname{dom} A), A$ is locally bounded at $x_{0}$. Hence there exists some $\rho>0$ and $K>0$ such that $A$ is bounded by $K$ on $\mathbb{B}\left(x_{0}, \rho\right)$. Let

$$
T^{*}:=(v+2 K)^{-1}(\rho / 3)>0 \text {, i.e. } v\left(T^{*}\right)+2 K T^{*}=\rho / 3,
$$

and

$$
\left.\lambda_{0}:=\frac{\rho}{3\left\|A^{0} x_{0}\right\|} \quad \text { if }\left\|A^{0} x_{0}\right\|=0 \text {, we set } \lambda_{0}:=+\infty\right) .
$$

Consider the sequence of functions $\left(x_{\lambda}\right)_{0<\lambda<\lambda_{0}}$ where $x_{\lambda}(\cdot)$ is the unique solution of the approximate sweeping process (12). Let

$$
T_{\lambda}=\sup \left\{\tau:\left\|x_{\lambda}(t)-x_{0}\right\| \leq \rho / 3, \quad \forall t \in[0, \tau]\right\}
$$

It is clear that $T_{\lambda}>0$. We will prove that $T_{\lambda} \geq T^{*}$ for all $\lambda<\lambda_{0}$. Suppose there exists some $\lambda<\lambda_{0}$ such that $T_{\lambda}<T^{*}$. From the definition of $T_{\lambda}$, one deduces that

$$
\left\|x_{\lambda}\left(T_{\lambda}\right)-x_{0}\right\|=\rho / 3
$$

Note that $A_{\lambda} x_{\lambda}(t) \in A\left(J_{\lambda} x_{\lambda}(t)\right)$ and $\left\|A_{\lambda} x_{\lambda}(t)\right\| \leq\left\|A_{\lambda} x_{0}\right\|+\left\|x_{\lambda}(t)-x_{0}\right\| / \lambda \leq\left\|A^{0} x_{0}\right\|+\rho /(3 \lambda)$ for all $t \in\left[0, T_{\lambda}\right]$ (since $A_{\lambda}$ is $1 / \lambda$-Lipschitz continuous). We have

$$
\begin{aligned}
\left\|J_{\lambda} x_{\lambda}(t)-x_{0}\right\| & \leq\left\|J_{\lambda} x_{\lambda}(t)-x_{\lambda}(t)\right\|+\left\|x_{\lambda}(t)-x_{0}\right\| \leq \lambda\left\|A_{\lambda} x_{\lambda}(t)\right\|+\rho / 3 \\
& \leq \lambda_{0}\left\|A^{0} x_{0}\right\|+2 \rho /(3) \leq \rho,
\end{aligned}
$$

for all $t \in\left[0, T_{\lambda}\right]$. Hence $J_{\lambda} x_{\lambda}(t) \in \mathbb{B}\left(x_{0}, \rho\right)$ and thus $\left\|A_{\lambda} x_{\lambda}(t)\right\| \leq K$ for all $t \in\left[0, T_{\lambda}\right]$. From (14), one has $\left\|\dot{x_{\lambda}}(t)\right\| \leq 2 K+k_{C}$ for a.e. $t \in\left[0, T_{\lambda}\right]$. Therefore,

$$
\left\|x_{\lambda}\left(T_{\lambda}\right)-x_{0}\right\| \leq \int_{0}^{T_{\lambda}}\left\|\dot{x_{\lambda}}(t)\right\| \mathrm{d} t<2 K T^{*}+v\left(T^{*}\right)=\rho / 3,
$$

which is a contradiction with (27). In conclusion, the sequence $\left(x_{\lambda}\right)_{0<\lambda<\lambda_{0}}$ satisfies for a.e. $t \in\left[0, T^{*}\right]$

$$
\left\{\begin{array}{l}
\left\|x_{\lambda}(t)-x_{0}\right\| \leq \rho / 3, \quad\left\|A_{\lambda} x_{\lambda}(t)\right\| \leq K, \\
\left\|\dot{x}_{\lambda}(t)+A_{\lambda} x_{\lambda}(t)\right\| \leq K+\dot{v}(t), \quad\left\|\dot{x}_{\lambda}(t)\right\| \leq 2 K+\dot{v}(t) .
\end{array}\right.
$$

Similarly to the proof of Theorem 3.2, there exists a solution $x(\cdot)$ of problem (1) on $\left[0, T^{*}\right]$ such that $\left\|x(t)-x_{0}\right\| \leq \rho / 3 \quad \forall t \in\left[0, T^{*}\right]$.

We prove that it is also the unique solution of $(1)$ on $\left[0, T^{*}\right]$. Let $y(\cdot)$ be another solution of (1) on $\left[0, T_{1}^{*}\right]$ for some positive $T_{1}^{*} \leq T^{*}$. It is sufficient to prove that $y(\cdot) \equiv x(\cdot)$ on $\left[0, T_{1}^{*}\right]$. Note that $A$ is bounded by $K$ on $\mathbb{B}\left(x_{0}, \rho\right)$. If $\left\|y(t)-x_{0}\right\| \leq \rho / 3, \quad \forall t \in\left[0, T_{1}^{*}\right]$, then similarly to the proof of Theorem 3.3, two solutions coincide on $\left[0, T_{1}^{*}\right]$. If there exists some $t \in\left[0, T_{1}^{*}\right)$ such that $\left\|y(t)-x_{0}\right\|>\rho / 3$ then there exists positive $T_{2}^{*}<T_{1}^{*}$ such that $\left\|y(t)-x_{0}\right\|<\rho / 3$ for all $t \in\left[0, T_{2}^{*}\right)$ and

$$
\left\|y\left(T_{2}^{*}\right)-x_{0}\right\|=\rho / 3
$$


Therefore, $y\left(\left[0, T_{2}^{*}\right]\right) \subset \mathbb{B}\left(x_{0}, \rho\right)$ and hence for all $t \in\left[0, T_{2}^{*}\right]$, one has $\|\dot{y}(t)\| \leq 2 K+\dot{v}(t)$. We deduce that

$$
\left\|y\left(T_{2}^{*}\right)-x_{0}\right\| \leq 2 K T_{2}^{*}+v\left(T_{2}^{*}\right)<2 K T^{*}+v\left(T^{*}\right)=\rho / 3,
$$

which is a contradiction with (29).

Remark 2: If $\operatorname{rge} C \subset \operatorname{int}(\operatorname{dom} A)$, then for a given initial condition $x_{0} \in C(0)$, one can define the unique solution on its maximal interval of existence (by Proposition 3.5). The solution can be extended globally for the particular case where $A=\partial \varphi$ with $\varphi: H \rightarrow \mathbb{R} \cup\{+\infty\}$ a proper lsc, convex function and $C(s) \subset C(t)$ for any $t \geq s \geq 0$, but $A^{0}$ is not necessarily satisfied the linear growth condition. In addition, the Lyapunov stability and some asymptotic behaviour of the unique solution can be obtained.

Theorem 3.6: Let the Assumptions 1, 2 hold. Consider the case $A=\partial \varphi$ where $\varphi: H \rightarrow \mathbb{R} \cup\{+\infty\}$ is a proper lsc convex function and $C(s) \subset C(t)$ for any $t \geq s \geq 0$. Suppose that $C(t) \subset \operatorname{int}(\operatorname{dom} \partial \varphi)$ for all $t \geq 0$. Then the differential inclusion

$$
\left\{\begin{array}{l}
\dot{x}(t) \in-\partial \varphi(x(t))-N_{C(t)}(x(t)) \text { a.e. } t \in[0,+\infty), \\
x(0)=x_{0} \in C(0)
\end{array}\right.
$$

has a unique global solution $x(\cdot)$ satisfying

$$
\frac{\mathrm{d}}{\mathrm{d} t} \varphi(x(t))+\|\dot{x}(t)\|^{2} \leq 0, \text { for a.e. } t \geq 0 .
$$

In particular, $\varphi$ is a Lyapunov function of the problem (30). Furthermore if $\varphi$ is bounded from below on $\operatorname{rge}(C)$ then $\varphi_{\infty}:=\lim _{t \rightarrow+\infty} \varphi(x(t))$ exists and $\dot{x} \in L^{2}([0,+\infty) ; H)$ with $\int_{0}^{+\infty}\|\dot{x}(s)\|^{2} \mathrm{~d} s \leq \varphi\left(x_{0}\right)-\varphi_{\infty}$.

Proof: It is clear that $\partial \varphi$ is a maximal monotone operator. From Proposition 3.5, there exists a unique solution of problem (30) defined on its maximal interval of existence $\left[0, T_{\max }\right)\left(0<T_{\max } \leq+\infty\right)$. There exists a mapping $\xi:\left[0, T_{\max }\right) \rightarrow H$ such that $\xi(t) \in \partial \varphi(x(t))$ and $\dot{x}(t) \in-\xi(t)-N_{C}(x(t))$. Since $\varphi$ is locally Lipschitz continuous on $\operatorname{rge}(C)$ (as $\partial \varphi$ is locally bounded on $\operatorname{rge}(C)$ ) and $x(\cdot)$ is locally absolutely continuous on $\left[0, T_{\max }\right)$, then $\varphi \circ x(\cdot)$ is differentiable a.e. on $\left[0, T_{\max }\right)$. Fix some $t \in\left(0, T_{\max }\right)$ at which $x(\cdot)$ and $\varphi \circ x(\cdot)$ are differentiable. By the convexity of $\varphi$ and the fact that $\xi(t) \in \partial \varphi(x(t))$, we get for every $\varepsilon>0$ that

$$
\left\{\begin{array}{l}
\frac{\varphi(x(t+\varepsilon))-\varphi(x(t))}{\varepsilon} \geq\left\langle\frac{x(t+\varepsilon)-x(t)}{\varepsilon}, \xi(t)\right\rangle, \\
\frac{\varphi(x(t-\varepsilon))-\varphi(x(t))}{-\varepsilon} \leq\left\langle\frac{x(t-\varepsilon)-x(t)}{-\varepsilon}, \xi(t)\right\rangle .
\end{array}\right.
$$

By letting $\varepsilon \rightarrow 0^{+}$, we obtain

On the other hand, we have

$$
\frac{\mathrm{d}}{\mathrm{d} t} \varphi(x(t))=\langle\dot{x}(t), \xi(t)\rangle
$$

$$
\dot{x}(t)+\xi(t) \in-N_{C(t)}(x(t)) .
$$

There exists some $\gamma>0$ such that

$$
\langle\dot{x}(t)+\xi(t), x(t)-c\rangle \leq \gamma\|c-x(t)\|^{2} \forall c \in C(t) .
$$

Taking $c=x(t-\varepsilon) \in C(t-\varepsilon) \subset C(t)$ in (34) for some $\varepsilon>0$. Dividing both sides of the inequality above by $\varepsilon$ and letting $\varepsilon \rightarrow 0^{+}$, one deduces that

$$
\langle\dot{x}(t)+\xi(t), \dot{x}(t)\rangle \leq 0
$$


From (33) and (35), we imply that

$$
\frac{\mathrm{d}}{\mathrm{d} t} \varphi(x(t))+\|\dot{x}(t)\|^{2} \leq 0
$$

We prove now that $T_{\max }=+\infty$. Suppose that $T_{\max }<+\infty$. Fix some $x \in C$ and let $\alpha=$ $\left\|\partial \varphi^{0}(x)\right\|, \beta=\left\|\varphi(x)-\left\langle\partial \varphi^{0}(x), x\right\rangle\right\|$. For every $y \in C$, we have

$$
\varphi(y) \geq \varphi(x)+\left\langle\partial \varphi^{0}(x), y-x\right\rangle \geq-\alpha\|y\|-\beta .
$$

By (36), for all $0 \leq s<t<T_{\max }$, one has

$$
\|x(t)-x(s)\|^{2} \leq(t-s) \int_{s}^{t}\|\dot{x}(s)\|^{2} \mathrm{~d} s \leq(t-s)(\varphi(x(s))-\varphi(x(t))) .
$$

By setting $s=0$ in (37), we obtain

$$
\left\|x(t)-x_{0}\right\|^{2} \leq t\left(\varphi\left(x_{0}\right)-\varphi(x(t))\right) \leq T_{\max }\left(\alpha\|x(t)\|+\beta+\varphi\left(x_{0}\right)\right) .
$$

Therefore $\sup _{t \in\left[0, T_{\max }\right)}\|x(t)\|<+\infty$ and hence $\sup _{t \in\left[0, T_{\max }\right)}(-\varphi(x(t)))<+\infty$. From (37), one obtains that $(x(t))_{t \in\left[0, T_{\max }\right.}$ is a Cauchy sequence in $H$. Hence $x_{\max }:=\lim _{t \rightarrow T_{\max }} x(t)$ exists and $x_{\max } \in C\left(T_{\max }\right)$. Using Proposition 3.5, there exist some $\delta>0$ and a unique solution of the differential inclusion

$$
\left\{\begin{array}{l}
\dot{y}(t) \in-\partial \varphi(y(t))-N_{C(t)}(y(t)) \text { a.e. } t \in\left[T_{\max }, T_{\max }+\delta\right] \\
y\left(T_{\max }\right)=x_{\max } \in C\left(T_{\max }\right) .
\end{array}\right.
$$

Define $z(\cdot) \equiv x(\cdot)$ on $\left[0, T_{\max }\right)$ and $z(\cdot) \equiv y(\cdot)$ on $\left[T_{\max }, T_{\max }+\delta\right]$. Then $z(\cdot)$ is the solution of (30) on $\left[0, T_{\max }+\delta\right]$, a contradiction. Hence $T_{\max }=+\infty$.

As a consequence, $\varphi \circ x(\cdot)$ is non-increasing on $\mathbb{R}_{+}$due to (36). Hence if $\varphi$ is bounded from below on $\operatorname{rge}(C)$ then $\lim _{t \rightarrow+\infty} \varphi(x(t))=\varphi_{\infty}$ exists and from (36), one has $\dot{x} \in L^{2}([0,+\infty) ; H)$ with $\int_{0}^{+\infty}\|\dot{x}(s)\|^{2} \mathrm{~d} s \leq \varphi\left(x_{0}\right)-\varphi_{\infty}$.

Example 3.7: Consider the non-regular electrical circuit described in Figure 2 of [5]. The evolution of the current $x(\cdot)$ through the load resistance $R>0$ is governed by

$$
\dot{x}(t)+\frac{R}{L} x(t) \in-N_{C(t)}(x(t)) \text { a.e. } t \geq 0, x(0)=x_{0} \text { with } C(t)=[c(t),+\infty),
$$

where $c(t)$ is a current source and $L>0$ is the inductance of an inductor. If $c(\cdot):[0,+\infty) \rightarrow \mathbb{R}$ is non-increasing absolutely continuous then all the assumptions in Theorem 3.6 are satisfied with $\varphi(x)=\frac{R}{2 L} x^{2}$. Then one obtains that the function $t \mapsto|x(t)|$ is non-increasing and the limit $\gamma:=\lim _{t \rightarrow+\infty}|x(t)|$ exists. In addition, one has

$$
\int_{0}^{+\infty}\|\dot{x}(s)\|^{2} \mathrm{~d} s \leq\left|x_{0}\right|-\gamma .
$$

Remark 3: In the case where $C(t) \equiv C \subset$ int.dom $A$ ) for all $t \geq 0$, it is sufficient to obtain the existence and uniqueness of solutions of our initial problem (1) if one of the following conditions holds: $A=\partial \varphi$ or $A^{0}$ is linearly bounded on $C$. 


\section{Some regular properties of solutions and non-smooth Lyapunov pairs}

In this section, in order to study the Lyapunov pairs of problem (1), we restrict ourself to the case where $C(\cdot) \equiv C$ does not depend on time. Lyapunov analysis of time dependent case is not an easy task due to the lack of regularity of the solutions. In addition, we suppose that $C \subset$ int.dom $A$ ) is closed, $r$-prox-regular and either $A^{0}$ is linearly bounded on $C$ or $A=\partial \varphi$ where $\varphi: H \rightarrow \mathbb{R} \cup\{+\infty\}$ be a proper lsc, convex function. From the previous section, for a given $x_{0} \in C$, the differential inclusion

$$
\left\{\begin{array}{l}
\dot{x}(t) \in-A x(t)-N_{C}(x(t)) \text { a.e. } t \in[0,+\infty), \\
x(0)=x_{0} \in C
\end{array}\right.
$$

has a unique solution $x(\cdot)$ defined on $[0,+\infty)$ which is locally absolutely continuous. We will show that $x(\cdot)$ is right-differentiable for all $t \geq 0$ and $\dot{x}^{+}(\cdot)$ is right-continuous. This result can be considered as a generalization of [20, Theorem 3.2], when $A$ is a single-valued and Lipschitz continuous function. In this context, the notion of weakly lower semi-continuous Lyapunov pair for problem (39) as well as the proximal, asymptotic subgradients play an important role in our analysis. The following lemmas will be useful.

Lemma 4.1: For all $x \in C$, the set $A(x)+N_{C}(x)$ is closed and convex.

Proof: Clearly, for all $x \in C$, the sets $A(x)$ and $N_{C}(x)$ are closed and convex (and thus weakly closed). Hence $A(x)+N_{C}(x)$ is convex. Let $z_{n}=x_{n}+y_{n} \in A(x)+N_{C}(x)$ such that $x_{n} \in A(x), y_{n} \in N_{C}(x)$ and $z_{n} \rightarrow z_{0}$ for some $z_{0} \in H$. Since $\left(x_{n}\right)$ is bounded, there exist a subsequence, still denoted by $\left(x_{n}\right)$ and some $x_{0} \in H$ such that $x_{n}$ converges weakly to $x_{0}$ in $H$. Since $A(x)$ is weakly closed, $x_{0} \in A(x)$. On the other hand $y_{n}=z_{n}-x_{n}$ converges weakly to $z_{0}-x_{0}=: y_{0} \in N_{C}(x)$. Thus $z_{0}=x_{0}+y_{0} \in A(x)+N_{C}(x)$ and the conclusion follows.

Let $x(\cdot)$ be the unique solution of (39) satisfying $x(0)=x_{0}$. Using the Lemma 4.1, one can define the function $v: \mathbb{R}_{+} \rightarrow H$ by $v(t):=\left(-A x(t)-N_{C}(x(t))\right)^{0}$ and $v_{0}:=v(0)=\left(-A x_{0}-N_{C}\left(x_{0}\right)\right)^{0}$.

Lemma 4.2: We have

$$
\left\|v_{0}\right\| \leq \liminf _{t \rightarrow 0^{+}}\|v(t)\| .
$$

Proof: If $\lim \inf _{t \rightarrow 0^{+}}\|v(t)\|=+\infty$ then the conclusion holds. If $\liminf _{t \rightarrow 0^{+}}\|v(t)\|=\alpha<+\infty$, then there exists a sequence $\left(t_{n}\right)_{n \geq 1}$ such that $t_{n} \rightarrow 0^{+}$and $\lim _{n \rightarrow+\infty}\left\|v\left(t_{n}\right)\right\|=\alpha$. In particular, the sequence $\left(v\left(t_{n}\right)\right)_{n \geq 1}$ is bounded hence there exist a subsequence $\left(v\left(t_{n_{k}}\right)\right)_{k \geq 1}$ and $\xi \in H$ such that $\left(v\left(t_{n_{k}}\right)\right)_{k \geq 1}$ converges weakly to $\xi$. Recall that

$$
v\left(t_{n_{k}}\right)=\left(-A x\left(t_{n_{k}}\right)-N_{C}\left(x\left(t_{n_{k}}\right)\right)\right)^{0}=\left(f\left(t_{n_{k}}\right)-N_{C}\left(x\left(t_{n_{k}}\right)\right)\right)^{0} \in f\left(t_{n_{k}}\right)-N_{C}\left(x\left(t_{n_{k}}\right)\right),
$$

for some $f\left(t_{n_{k}}\right) \in-A x\left(t_{n_{k}}\right)$. Hence $f\left(t_{n_{k}}\right)-v\left(t_{n_{k}}\right) \in N\left(C ; x\left(t_{n_{k}}\right)\right)$. The sequence $\left(f\left(t_{n_{k}}\right)\right)_{k}$ is bounded for $k$ large enough since $A$ is locally bounded hence there exist a subsequence, still denoted itself and some $\gamma \in H$ such that $f\left(t_{n_{k}}\right)$ converges weakly to $\gamma$ in $H$. Due to the closed-graph property of $A$, one must have $\gamma \in-A x_{0}$. On the other hand, we can find some $\beta>0$ such that $\left\|f\left(t_{n_{k}}\right)-v\left(t_{n_{k}}\right)\right\| \leq \beta$ for all $k \geq 1$. Using the prox-regularity of $C$, one has

$$
\left\langle f\left(t_{n_{k}}\right)-v\left(t_{n_{k}}\right), c-x\left(t_{n_{k}}\right)\right\rangle \leq \frac{\beta}{2 r}\left\|c-x\left(t_{n_{k}}\right)\right\|^{2} \quad \text { for all } c \in C, k \geq 1 .
$$

Let $k \rightarrow+\infty$, we get

$$
\left\langle\gamma-\xi, c-x_{0}\right\rangle \leq \frac{\beta}{2 r}\left\|c-x_{0}\right\|^{2} \text { for all } c \in C
$$


Thus $\gamma-\xi \in N_{C}\left(x_{0}\right)$ or equivalently $\xi \in \gamma-N_{C}\left(x_{0}\right) \in-A x_{0}-N_{C}\left(x_{0}\right)$. Therefore,

$$
\left\|v_{0}\right\| \leq\|\xi\| \leq \liminf _{k \rightarrow+\infty}\left\|v\left(t_{n_{k}}\right)\right\|=\lim _{n \rightarrow+\infty}\left\|v\left(t_{n}\right)\right\|=\alpha
$$

due to the weak lower semi-continuity of the norm and the conclusion follows.

Lemma 4.3: Let $x(\cdot)$ be the unique solution of (39) satisfying $x(0)=x_{0}$. Then one has

$$
\limsup _{t \rightarrow 0^{+}}\left\|\frac{x(t)-x_{0}}{t}\right\| \leq\left\|v_{0}\right\|
$$

where $v_{0}=\left(-A x_{0}-N_{C}\left(x_{0}\right)\right)^{0}$.

Proof: Since $A$ is locally bounded on $C$, there exist some $\rho>0$ and $K>0$ such that $A$ is bounded by $K$ on $\mathbb{B}\left(x_{0}, \rho\right)$. Note that $x(\cdot)$ is continuous, there exists some positive $T$ such that $x([0, T]) \subset \mathbb{B}\left(x_{0}, \rho\right)$. We have

$$
\left\{\begin{array}{l}
\dot{x}(t)-f(t) \in-N_{C}(x(t)) \text { a.e. } t \in[0, T] \\
v_{0}-f_{0} \in-N_{C}\left(x_{0}\right)
\end{array}\right.
$$

for some $f_{0} \in-A x_{0}, f(t) \in-A x(t)$ and $\|\dot{x}(t)-f(t)\| \leq\|f(t)\| \leq K$ for a.e. $t \geq 0$. Using the prox-regularity of $C$ and the monotonicity of $A$, one has

$$
\left\langle\dot{x}(t)-v_{0}, x(t)-x_{0}\right\rangle \leq \frac{K}{r}\left\|x(t)-x_{0}\right\|^{2},
$$

which implies that

$$
\frac{1}{2} \frac{\mathrm{d}}{\mathrm{d} t}\left\|x(t)-x_{0}\right\|^{2} \leq\left\|v_{0}\right\|\left\|x(t)-x_{0}\right\|+\frac{K}{r}\left\|x(t)-x_{0}\right\|^{2}
$$

Using Gronwall's inequality (Lemma 2.4), one obtains for all $t \in[0, T]$ that

$$
\left\|x(t)-x_{0}\right\| \leq\left\|v_{0}\right\| \int_{0}^{t} e^{\frac{K}{r}(t-s)} \mathrm{d} s=\left\|v_{0}\right\| \int_{0}^{t} e^{\frac{K s}{r}} \mathrm{~d} s .
$$

Hence,

$$
\limsup _{t \rightarrow 0^{+}}\left\|\frac{x(t)-x_{0}}{t}\right\| \leq\left\|v_{0}\right\| \lim _{t \rightarrow 0^{+}} \frac{1}{t} \int_{0}^{t} e^{\frac{K s}{r}} \mathrm{~d} s=\left\|v_{0}\right\| .
$$

The proof is completed.

Lemma 4.4: $\quad$ There exist some $K>0, T>0$ such that for a.e. $t \in[0, T]$, one has

$$
\|\dot{x}(t)\| \leq\left\|v_{0}\right\| e^{K t / r} .
$$

Proof: There exist some $\rho>0$ and $K>0$ such that $A$ is bounded by $K$ on $\mathbb{B}\left(x_{0}, \rho\right)$. Let $x(\cdot), y(\cdot)$ be the unique solution of (39) satisfying initial conditions $x(0)=x_{0}, y(0)=x(h)$, respectively, where $h$ is small enough such that there exist some positive $T$ and $x([0, T]), y([0, T]) \subset \mathbb{B}\left(x_{0}, \rho\right)$. Similarly as in Theorem 3.3, with $k_{C}=0$, one has

$$
\|x(t)-y(t)\| \leq\left\|x_{0}-y_{0}\right\| e^{K t / r}, \quad \text { for all } t \in[0, T] .
$$


Note that $y(t)=x(t+h)$ for all $t \geq 0$. From (50), we deduce that

$$
\left\|\frac{x(t+h)-x(t)}{h}\right\| \leq\left\|\frac{x(h)-x(0)}{h}\right\| e^{K t / r}, \quad \text { for all } t \in[0, T]
$$

Fix some $t \in[0, T]$ such that $\dot{x}(t)$ exists. Taking the limsup in both sides of (51) as $h \rightarrow 0^{+}$and using Lemma 4.3, one gets

$$
\|\dot{x}(t)\| \leq\left\|v_{0}\right\| e^{K t / r}
$$

Thus (49) follows.

Now we are ready to formulate the regular properties of the differential inclusion (39).

Theorem 4.5: Let $x(\cdot)$ be the unique solution of problem (39) satisfying $x(0)=x_{0}$. Then we have

(i) $\dot{x}(t)=v(t)=\left(-A x(t)-N_{C}(x(t))\right)^{0}$ for a.e. $t \in[0,+\infty)$.

(ii) For all $t^{*} \in[0,+\infty)$, the right derivative $\dot{x}^{+}\left(t^{*}\right)$ exists and satisfies

$$
\dot{x}^{+}\left(t^{*}\right)=\left(-A x\left(t^{*}\right)-N_{C}\left(x\left(t^{*}\right)\right)\right)^{0} .
$$

Furthermore $\dot{x}^{+}(\cdot)$ is right-continuous on $[0,+\infty)$.

Proof: Let $E=\{t \in[0,+\infty): \dot{x}(t)$ exists $\}$. It is clear that the Lebesgue measure of $[0,+\infty) \backslash E$ is zero.

(i) Fix $t^{*} \in E$. Let $y(\cdot)$ be the unique solution of problem (39) with initial condition $y(0)=x\left(t^{*}\right)$. Then $y(t)=x\left(t+t^{*}\right)$ for all $t \geq 0$. Applying Lemma 4.3, we get

$$
\underset{t \rightarrow 0^{+}}{\limsup }\left\|\frac{y(t)-y(0)}{t}\right\| \leq\left\|\left(-A y(0)-N_{C}(y(0))\right)^{0}\right\|,
$$

or equivalently,

$$
\limsup _{t \rightarrow 0^{+}}\left\|\frac{x\left(t+t^{*}\right)-x\left(t^{*}\right)}{t}\right\| \leq\left\|\left(-A x\left(t^{*}\right)-N_{C}\left(x\left(t^{*}\right)\right)\right)^{0}\right\| .
$$

Hence

$$
\left\|\dot{x}\left(t^{*}\right)\right\| \leq\left\|\left(-A x\left(t^{*}\right)-N_{C}\left(x\left(t^{*}\right)\right)\right)^{0}\right\| .
$$

On the other hand $\dot{x}\left(t^{*}\right) \in-A x\left(t^{*}\right)-N_{C}\left(x\left(t^{*}\right)\right)$, thus $\dot{x}\left(t^{*}\right)=\left(-A x\left(t^{*}\right)-N_{C}\left(x\left(t^{*}\right)\right)\right)^{0}$.

(ii) It is sufficient to prove this property for $t^{*}=0$. Using (i) and Lemma 4.4, there exist some $K>0, T>0$ such that for all $t \in[0, T] \cap E$, we have

$$
\|v(t)\| \leq\left\|v_{0}\right\| e^{K t / r},
$$

where $v(t)=\left(-A x(t)-N_{C}(x(t))\right)^{0}$. It follows from (55) that

$$
\limsup _{t \rightarrow 0^{+}, t \in E}\|v(t)\| \leq\left\|v_{0}\right\|
$$

On the other hand, from Lemma 4.2 we deduce that

$$
\left\|v_{0}\right\| \leq \liminf _{t \rightarrow 0^{+}}\|v(t)\| \leq \liminf _{t \rightarrow 0^{+}, t \in E}\|v(t)\| .
$$


From (56) and (57), we obtain

$$
\lim _{t \rightarrow 0^{+}, t \in E}\|v(t)\|=\left\|v_{0}\right\|
$$

Thus for any sequence $\left(t_{n}\right)_{n \geq 1} \subset E$ such that $t_{n} \rightarrow 0^{+}$, we have

$$
\left\|v\left(t_{n}\right)\right\| \rightarrow\left\|v_{0}\right\| \text { as } n \rightarrow+\infty
$$

Then $\left(v\left(t_{n}\right)\right)_{n>1}$ is bounded and therefore there exist some $v^{*} \in H$ and a subsequence $\left(v\left(t_{n_{k}}\right)\right)_{k \geq 1}$ such that $v\left(t_{n_{k}}\right)$ converges weakly to $v^{*}$ as $k \rightarrow+\infty$. Similarly as in Lemma 4.2, we can prove that $v^{*} \in-A x_{0}-N_{C}\left(x_{0}\right)$. On the other hand, thanks to (59), we have

$$
\left\|v^{*}\right\| \leq \liminf _{k \rightarrow+\infty}\left\|v\left(t_{n_{k}}\right)\right\|=\lim _{n \rightarrow+\infty}\left\|v\left(t_{n}\right)\right\|=\left\|v_{0}\right\|
$$

By the definition of $v_{0}$, we obtain $v^{*}=v_{0}$ and the set of weak cluster point of $\left(v\left(t_{n}\right)\right)_{n>1}$ contains only $v_{0}$. Consequently $v\left(t_{n}\right)$ converges weakly to $v_{0}$. Taking into account (59), one deduces that $v\left(t_{n}\right)$ converges strongly to $v_{0}$. In conclusion, we get

$$
\lim _{t \rightarrow 0^{+}, t \in E} v(t)=v_{0}
$$

Due to the absolute continuity of $x(\cdot)$ and (i) in Theorem 4.5, for all $h>0$, we have

$$
x(h)-x_{0}=\int_{0}^{h} \dot{x}(s) \mathrm{d} s=\int_{0}^{h} v(s) \mathrm{d} s,
$$

where $v(\cdot)$ is locally integrable and satisfying (61). Now we prove that

$$
\lim _{h \rightarrow 0^{+}} \frac{1}{h} \int_{0}^{h} v(s) \mathrm{d} s=v_{0} .
$$

Let $\epsilon>0$ be given. From (61), there exists $\delta>0$ such that for all $s \in E, s \leq \delta$, we have $\left\|v(s)-v_{0}\right\| \leq \epsilon$. Hence, for all $h \leq \delta$, one gets

$$
\begin{aligned}
\left\|\frac{1}{h} \int_{0}^{h} v(s) \mathrm{d} s-v_{0}\right\| & \leq \frac{1}{h} \int_{0}^{h}\left\|v(s)-v_{0}\right\| \mathrm{d} s \\
& =\frac{1}{h} \int_{[0, h] \cap E}\left\|v(s)-v_{0}\right\| \mathrm{d} s \\
& \leq \frac{\epsilon}{h} \int_{[0, h] \cap E} \mathrm{~d} s=\epsilon .
\end{aligned}
$$

Therefore (63) is proved. From (62), the right derivative $\dot{x}^{+}(0)$ exists and satisfies

$$
\dot{x}^{+}(0)=v_{0}=\left(-A x_{0}-N_{C}\left(x_{0}\right)\right)^{0} .
$$

Then taking the limit in both sides of (51) as $h \rightarrow 0^{+}$, we deduce that for all $t \geq 0$ :

$$
\left\|\dot{x}^{+}(t)\right\| \leq\left\|\dot{x}^{+}(0)\right\| e^{K t / r},
$$

or equivalently,

$$
\|v(t)\| \leq\left\|v_{0}\right\| e^{K t / r} .
$$


Therefore

$$
\limsup _{t \rightarrow 0^{+}}\|v(t)\| \leq\left\|v_{0}\right\| \text {. }
$$

Using (57), we obtain $\lim _{t \rightarrow 0^{+}}\|v(t)\|=\left\|v_{0}\right\|$. Similarly as in (61), we can prove that $\lim _{t \rightarrow 0^{+}} v(t)=v_{0}$, which means that $\dot{x}^{+}(\cdot)$ is right-continuous at 0 . The proof of Theorem 4.5 is completed.

Next, let us consider the non-smooth lower semi-continuous Lyapunov functions for problem (39). First, we recall the definition of a Lyapunov pair, as in [19]. Denote by

$$
\Gamma_{w}(H):=\{\varphi: H \rightarrow \mathbb{R} \cup\{+\infty\} \mid \varphi \text { is proper and weakly lsc }\} \text {, }
$$

and

$$
\Gamma_{+}(H):=\left\{\varphi: H \rightarrow \mathbb{R}_{+} \cup\{+\infty\} \mid \varphi \text { is proper and lsc }\right\} .
$$

Definition 3: Let $V \in \Gamma_{w}(H), W \in \Gamma_{+}(H)$ and $a \geq 0$. We say that $(V, W)$ is an $a$-Lyapunov pair for problem (39) if for all $x_{0} \in C$, we have

$$
e^{a t} V\left(x\left(t ; x_{0}\right)\right)+\int_{0}^{t} W\left(x\left(\tau ; x_{0}\right)\right) \mathrm{d} \tau \leq V\left(x_{0}\right) \text { for all } t \geq 0,
$$

where $x\left(t ; x_{0}\right)$ denotes the unique solution of problem (39) starting at $x_{0}$. If $a=0$, then $(V, W)$ is called a Lyapunov pair. In addition, $V$ is called a Lyapunov function if $W=0$.

We have the following theorem which gives necessary and sufficient conditions of a Lyapunov pair. Note that, since $A$ is locally bounded on $C$, we do not need to check for the singular subdifferentials as in [19].

Theorem 4.6: Let $V \in \Gamma_{w}(H), W \in \Gamma_{+}(H), a \geq 0$ and dom $V \subset C$. Then the following assertions are equivalent:

(i) For all $x_{0} \in \operatorname{dom} V$, we have

$$
e^{a t} V\left(x\left(t ; x_{0}\right)\right)+\int_{0}^{t} W\left(x\left(\tau ; x_{0}\right)\right) \mathrm{d} \tau \leq V\left(x_{0}\right) \quad \forall t \geq 0 .
$$

(ii) For all $x_{0} \in \operatorname{dom} V$, there exists $\rho\left(x_{0}\right)>0$ such that

$$
e^{a t} V\left(x\left(t ; x_{0}\right)\right)+\int_{0}^{t} W\left(x\left(\tau ; x_{0}\right)\right) \mathrm{d} \tau \leq V\left(x_{0}\right) \forall t \in\left[0, \rho\left(x_{0}\right)\right] .
$$

(iii) For all $y \in C$, we have

$$
\sup _{\xi \in \partial_{P} V(y)}\left\langle\xi,\left(-A y-N_{C}(y)\right)^{0}\right\rangle+a V(y)+W(y) \leq 0 .
$$

(iv) For all $y \in C$, we have

$$
\left\{\begin{array}{l}
\sup _{\xi \in \partial_{P} V(y)} \min _{y^{*} \in\left(-A y-N_{C}(y)\right) \cap \mathbb{B}(0,\|A y\|)}\left\langle\xi, y^{*}\right\rangle+a V(y)+W(y) \leq 0, \\
\sup _{\xi \in \partial_{\infty} V(y)} \min _{y^{*} \in\left(-A y-N_{C}(y)\right) \cap \mathbb{B}(0,\|A y\|)}\left\langle\xi, y^{*}\right\rangle \leq 0 .
\end{array}\right.
$$


Proof: Without loss of generality, suppose that $W$ is Lipschitz continuous on bounded sets (see [19, Lemma 3.1] or [14]). The plan of the proof is the following:

(i) $\Leftrightarrow$ (ii) and (ii) $\Rightarrow$ (iii) $\Rightarrow$ (iv) $\Rightarrow$ (ii).

(i) $\Leftrightarrow$ (ii): see Proposition 3.2 [19];

(ii) $\Rightarrow$ (iii) : Let $y \in C, \xi \in \partial_{P} V(y)$. Then $y \in \operatorname{dom} V$ and $(\xi,-1) \in N_{\text {epi } V}^{P}(y, V(y))$. From (ii), one infers that

$$
\left(x(t ; y), e^{-a t} V(y)-e^{-a t} \int_{0}^{t} W(x(\tau ; y)) \mathrm{d} \tau\right) \in \text { epi } V \quad \forall t \in[0, \rho(y)]
$$

By the definition of $N_{\text {epi } V}^{P}(y, V(y))$, there exists $\beta>0$ such that for all $t \in[0, \rho(y)]$, one has

$$
\begin{aligned}
& \left\langle(\xi,-1),\left(x(t ; y), e^{-a t} V(y)-e^{-a t} \int_{0}^{t} W(x(\tau ; y)) \mathrm{d} \tau\right)-(y, V(y))\right\rangle \\
\leq & \beta\left\|\left(x(t ; y), e^{-a t} V(y)-e^{-a t} \int_{0}^{t} W(x(\tau ; y)) \mathrm{d} \tau\right)-(y, V(y))\right\|^{2},
\end{aligned}
$$

which is equivalent to

$$
\langle\xi, x(t ; y)-y\rangle-m(t) \leq \beta\|x(t ; y)-y\|^{2}+\beta m^{2}(t),
$$

where

$$
m(t):=\left(e^{-a t}-1\right) V(y)-e^{-a t} \int_{0}^{t} W(x(\tau ; y)) \mathrm{d} \tau .
$$

Dividing both sides of (68) by $t>0$, letting $t \rightarrow 0^{+}$and taking into account that $\dot{x}^{+}(0 ; y)=$ $\left(-A y-N_{C}(y)\right)^{0}$, one obtains

$$
\left\langle\xi,\left(-A y-N_{C}(y)\right)^{0}\right\rangle+a V(y)+W(y) \leq 0 .
$$

(iii) $\Rightarrow$ (iv) : Obviously, one has $\left(-A y-N_{C}(y)\right)^{0} \in\left(-A y-N_{C}(y)\right) \cap \mathbb{B}(0,\|A y\|)$. Thus, (iii) implies the first inequality of (iv). It remains to check the second inequality of (iv). Let $\xi \in \partial_{\infty} V(y)$. Thanks to [17], as we discussed in Section 2, there exist sequences $\left(\alpha_{k}\right)_{k \in \mathbb{N}} \subset \mathbb{R}_{+},\left(y_{k}\right)_{k \in \mathbb{N}},\left(\xi_{k}\right)_{k \in \mathbb{N}}$ such that $\alpha_{k} \rightarrow 0^{+}, y_{k} \rightarrow_{\varphi} y, \xi_{k} \in \partial_{P} \varphi\left(y_{k}\right)$ and $\alpha_{k} \xi_{k} \rightarrow \xi$. For each $k$, one can find $y_{k}^{*} \in$ $\left(-A y-N_{C}(y)\right) \cap \mathbb{B}(0,\|A y\|)$ such that

$$
\left\langle\xi_{k}, y_{k}^{*}\right\rangle+a V\left(y_{k}\right)+W\left(y_{k}\right) \leq 0
$$

Since the sequence $\left(y_{k}^{*}\right)$ is bounded, one can extract a subsequence, without relabelling, and some $y^{*} \in\left(-A y-N_{C}(y)\right) \cap \mathbb{B}(0,\|A y\|)$ such that $y_{k}^{*} \rightarrow y^{*}$ weakly. Multiplying both sides of (69) by $\alpha_{k}$ and let $k \rightarrow+\infty$ then one obtains that

$$
\left\langle\xi, y^{*}\right\rangle \leq 0
$$

which implies the second inequality of (iv).

(iv) $\Rightarrow$ (ii) : Let $x_{0} \in \operatorname{dom} V$. Since $A$ is locally bounded at $x_{0}$, there exist $\varepsilon>0$ and $K>0$ such that $A$ is bounded by $K>0$ on $\mathbb{B}\left(x_{0}, 2 \varepsilon\right)$. On the other hand we can find some $T>0$ such that

$$
2\left\|x\left(t ; x_{0}\right)-x_{0}\right\|+\left|\left(e^{-a t}-1\right) V\left(x_{0}\right)-\int_{0}^{t} W\left(x\left(\tau ; x_{0}\right)\right) \mathrm{d} \tau\right|<\varepsilon, \quad \forall t \in[0, T] .
$$


In particular, $A\left(x\left([0, T] ; x_{0}\right)\right)$ is bounded by $K$. Define functions $h:[0, T] \rightarrow \mathbb{R}_{+}, \gamma:[0, T] \rightarrow \mathbb{R}$, $z_{n}:[0, T] \rightarrow H \times \mathbb{R}$ and $\eta:[0, T] \rightarrow \mathbb{R}_{+}$as follow

$$
\begin{gathered}
h(t):=\int_{0}^{t} W\left(x\left(\tau ; x_{0}\right)\right) \mathrm{d} \tau, \quad \gamma(t):=e^{-a t}\left(V\left(x_{0}\right)-h(t)\right), \\
z(t):=\left(x\left(t ; x_{0}\right), \gamma(t)\right), \quad \eta(t):=\frac{1}{2} d^{2}(z(t), \text { epi } V) .
\end{gathered}
$$

As in [19], $\eta$ is Lipschitz continuous on every compact interval in $(0, T)$ and for all $t \in(0, T)$, one has

$$
\partial_{C} \eta(t)=d(z(t) \text {, epi } V) \partial_{C} d(z(\cdot) \text {, epi } V)(t) \neq \emptyset,
$$

where $\partial_{C}$ denotes the Clarke subdifferential. We have then an estimation of $\partial_{C} \eta$ as in Lemma 4.7. Let $0<s<t<T$. By using Gronwall's inequality one has

$$
e^{-M t} \eta(t) \leq e^{-M s} \eta(s),
$$

where $M$ is defined in Lemma 4.7. Let $s \rightarrow 0$ then one has $d(z(t)$, epi $V)=0$ which implies that

$$
e^{a t} V\left(x\left(t ; x_{0}\right)\right)+\int_{0}^{t} W\left(x\left(\tau ; x_{0}\right)\right) \mathrm{d} \tau \leq V\left(x_{0}\right) .
$$

Since it is true for all $t \in[0, T]$, one obtains $i$ ) with $\rho\left(x_{0}\right)=T$.

The following technical lemma is useful for the proof of Theorem 4.6.

Lemma 4.7: There exist $M>0$ such that for almost all $t \in(0, T)$, one has

$$
\partial_{C} \eta(t) \subset(-\infty, M \eta(t)]
$$

Proof: Let $t \in(0, T)$ such that $x\left(\cdot ; x_{0}\right)$ is differentiable at $t$. If $z(t) \in$ epi $V$, then $\partial_{C} \eta(t)=\{0\}$ and the conclusion holds. Otherwise, assume that $z(t) \notin$ epi $V$. By using [19, Lemma A.3] and noting that $\left\|\dot{x}\left(t ; x_{0}\right)\right\|=\left\|\left(-A x\left(t ; x_{0}\right)-N_{C}\left(x\left(t ; x_{0}\right)\right)\right)^{0}\right\| \leq K$, we obtain

$$
\partial_{C} \eta(t) \subset \overline{\mathrm{co}}\left[\bigcup_{(u, \mu) \in \mathcal{M}}\left\langle z(t)-\left(\begin{array}{l}
u \\
\mu
\end{array}\right),\left(\begin{array}{c}
\left.-A x\left(t ; x_{0}\right)-N_{C}\left(x\left(t ; x_{0}\right)\right)\right) \cap K \mathbb{B} \\
-a \gamma(t)-e^{-a t} W\left(x\left(t ; x_{0}\right)\right)
\end{array}\right)\right],\right.
$$

where $\mathcal{M}:=\operatorname{Proj}(z(t)$, epi $V) \cap\left(\mathbb{B}\left(x_{0}, \varepsilon\right) \times[\gamma(t), \gamma(t)+\varepsilon]\right)$. Then it is sufficient to prove that for all $(u, \mu) \in \mathcal{M}$ and $\forall x \in\left(-A x\left(t ; x_{0}\right)-N_{C}\left(x\left(t ; x_{0}\right)\right)\right) \cap K \mathbb{B}$, we have

$$
\left\langle z(t)-\left(\begin{array}{l}
u \\
\mu
\end{array}\right),\left(\begin{array}{c}
x \\
-a \gamma(t)-e^{-a t} W\left(x\left(t ; x_{0}\right)\right)
\end{array}\right)\right\rangle \leq M \eta(t),
$$

for some $M>0$. Since $(u, \mu) \in \operatorname{Proj}(z(t)$, epi $V)$, the vector $z(t)-(u, \mu)=\left(x\left(t ; x_{0}\right)-u, \gamma(t)-\mu\right) \in$ $N_{\text {epi } V}^{P}(u, \mu)$ and $u \in \operatorname{dom} V \subset C$. We have $\gamma(t)-\mu \leq 0$. If $\gamma(t)-\mu=0$ then $x\left(t ; x_{0}\right)-u \in \partial_{\infty} V(u)$ and if $\gamma(t)-\mu<0$ then $\frac{x\left(t ; x_{0}\right)-u}{\mu-\gamma(t)} \in \partial_{P} V(u)$. From $\left.i v\right)$, there exists $u^{*} \in\left(-A u-N_{C}(u)\right) \cap \mathbb{B}(0,\|A u\|)$ such that

$$
\left\langle x\left(t ; x_{0}\right)-u, u^{*}\right\rangle \leq(\gamma(t)-\mu)(a V(u)+W(u)) .
$$


Note that $u \in \mathbb{B}\left(x_{0}, \varepsilon\right)$, hence $\left\|u^{*}\right\| \leq\|A u\| \leq\left\|A\left(\mathbb{B}\left(x_{0}, 2 \varepsilon\right)\right)\right\| \leq K$. We can write $x=x_{A}+x_{N}$ and $u^{*}=u_{A}^{*}+u_{N}^{*}$ such that $x_{A} \in-A x\left(t ; x_{0}\right), u_{A}^{*} \in-A u, x_{N} \in-N_{C}\left(x\left(t ; x_{0}\right)\right)$ and $u_{N}^{*} \in-N_{C}(u)$. Therefore $\left\|x_{N}\right\| \leq\|x\|+\left\|x_{A}\right\| \leq 2 K,\left\|u_{N}^{*}\right\| \leq\left\|u^{*}\right\|+\left\|u_{A}^{*}\right\| \leq 2 K$. Let $\beta:=\frac{2 K}{r}$ (recall that $C$ is $r$-prox-regular). Thanks to the maximality of $A$, the prox-regularity of $C$ and (74), one has

$$
\begin{aligned}
\left\langle x\left(t ; x_{0}\right)-u, x\right\rangle & =\left\langle x\left(t ; x_{0}\right)-u, x_{A}+x_{N}\right\rangle \\
& =\left\langle x\left(t ; x_{0}\right)-u, x_{A}-u_{A}^{*}+x_{N}-u_{N}^{*}\right\rangle+\left\langle x\left(t ; x_{0}\right)-u, u^{*}\right\rangle \\
& \leq \beta\left\|x\left(t ; x_{0}\right)-u\right\|^{2}+(\gamma(t)-\mu)(a V(u)+W(u)) .
\end{aligned}
$$

Note that we already have $\gamma(t)-\mu \leq 0$. If $\gamma(t)-\mu<0$ and suppose that $V(u) \leq \gamma(t)$. One obtains a contradiction

$$
d(z(t), \text { epi } V) \leq d(z(t),(u, \gamma(t)))<d(z(t),(u, \mu))=d(z(t), \text { epi } V)
$$

Hence if $\gamma(t)-\mu<0$, we have $V(u)>\gamma(t)$. Therefore,

$$
(\mu-\gamma(t))(\gamma(t)-V(u)) \leq 0
$$

Consequently,

$$
\begin{aligned}
& \left\langle x\left(t ; x_{0}\right)-u, x\right\rangle+a(\mu-\gamma(t)) \gamma(t)+(\mu-\gamma(t)) e^{-a t} W\left(x\left(t ; x_{0}\right)\right) \\
& \quad \leq \beta\left\|x\left(t ; x_{0}\right)-u\right\|^{2}+(\gamma(t)-\mu)(a V(u)+W(u))+a(\mu-\gamma(t)) \gamma(t) \\
& \quad+(\mu-\gamma(t)) e^{-a t} W\left(x\left(t ; x_{0}\right)\right) \\
& \quad \leq \beta\left\|x\left(t ; x_{0}\right)-u\right\|^{2}+a(\mu-\gamma(t))(\gamma(t)-V(u))+(\mu-\gamma(t))\left(W\left(x\left(t ; x_{0}\right)\right)-W(u)\right) \\
& \quad \leq \beta\left\|x\left(t ; x_{0}\right)-u\right\|^{2}+L_{W}|\mu-\gamma(t)|\left\|x\left(t ; x_{0}\right)-u\right\| \leq\left(\frac{L_{W}}{2}+\beta\right) \eta(t),
\end{aligned}
$$

where $L_{W}$ is the Lipschitz constant of $W$ on the ball $\mathbb{B}\left(x_{0}, \max \{\varepsilon, K\}\right)$. Therefore Lemma 4.7 holds with $M=\frac{L_{W}}{2}+\beta$.

\section{Conclusion}

By using a regularization technique, we study in this paper the existence and the uniqueness properties of a new variant of non-convex sweeping processes involving maximal monotone operators. Some regular properties of the solutions are refined when the moving set is fixed. It is showed that the unique solution is right-differentiable at any $t \geq 0$ and its right-derivative is right-continuous. Nonsmooth Lyapunov pair for such system is also considered by using the proximal and asymptotic subgradients. The Lyapunov analysis of the sweeping process (1) is an open question and is not an easy task due to the lack of regularity of the solutions.

\section{Disclosure statement}

No potential conflict of interest was reported by the authors.

\section{References}

[1] Moreau JJ. Proximité et dualité dans un espace hilbertien. Bull Soc Math France. 1965;93:273-299.

[2] Moreau JJ. Sur l'evolution d'un système élastoplastique. C R Acad Sci Paris Sér A-B. 1971;273:A118-A121.

[3] Moreau JJ. Rafle par un convexe variable I. Sém Anal Convexe Montpellier. 1971, Exposé 15.

[4] Moreau JJ. Rafle par un convexe variable II. Sém Anal Convexe Montpellier. 1972, Exposé 3. 
[5] Adly S, Haddad T, Thibault L. Convex Sweeping process in the framework of measure differential inclusions and evolution variational inequalities. Math Program Ser B. 2014;148:5-47.

[6] Edmond JF, Thibault L. Relaxation of an optimal control problem involving a perturbed sweeping process. Math Prog Ser B. 2005;104:347-373.

[7] Kunze M, Monteiro Marques MDP. An introduction to Moreau's sweeping process. In: Brogliato B, editor. Impacts in mechanical systems. Analysis and modelling. Berlin: Springer; 2000. p. 1-60.

[8] Mazade M, Thibault L. Regularization of differential variational inequalities with locally prox regular sets. Math Prog Ser B. 2013;139:243-269.

[9] Thibault L. Sweeping process with regular and nonregular sets. J Differ Equ. 2003;193:1-23.

[10] Thibault L. Regularization of nonconvex sweeping process in Hilbert space. Set-valued Anal. 2008;16:319-333.

[11] Rockafellar RT. On the maximality of the sum of nonlinear monotone operators. Trans Amer Math Soc. 1970;149:75-88.

[12] Brezis H. Opérateurs maximaux monotones et semi-groupes de contractions dans les espaces de Hilbert. Vol. 5, Mathematics Studies. Amsterdam: North-Holland American Elsevier; 1973.

[13] Marcellin S, Thibault L. Evolution problems associated with primal lower nice functions. J Convex Anal. 2006;2:385-421.

[14] Clarke FH, Ledyaev YS, Stern RJ, Wolenski PR. Nonsmooth analysis and control theory. Vol. 178, Graduate texts in mathematics. New York (NY): Springer; 1998.

[15] Rockafellar RT, Wets RB. Variational analysis. Berlin: Springer; 1998.

[16] Poliquin RA, Rockafellar RT, Thibault L. Local differentiability of distance functions. Trans Amer Math Soc. 2000;352:5231-5249.

[17] Mordukhovich B. Variational analysis and generalized differentiation I. Berlin: Springer-Verlag; 2006.

[18] Showalter RE. Monotone operators in Banach spaces and nonlinear partial differential equations. Providence (RI): American Mathematical Society; 1997.

[19] Adly S, Hantoute A, Thera M. Nonsmooth Lyapunov pairs for infinite-dimensional first-order differential inclusions. Nonlinear Anal. 2012;75:985-1008.

[20] Le BK. On properties of differential inclusions with prox-regular sets. Pac J Optim. 2017;13:17-27. 